\title{
THE
}

\section{Evaluation of Front Detection Methods for Satellite-Derived SST Data Using In Situ Observations}

\author{
David S. Ullman \\ University of Rhode Island, dullman@uri.edu \\ Peter C. Cornillon \\ University of Rhode Island, pcornillon@uri.edu
}

Follow this and additional works at: https://digitalcommons.uri.edu/gsofacpubs

Terms of Use

All rights reserved under copyright.

\footnotetext{
Citation/Publisher Attribution

Ullman, D.S. and P.C. Cornillon, 2000: Evaluation of Front Detection Methods for Satellite-Derived SST Data Using In Situ Observations. J. Atmos. Oceanic Technol., 17, 1667-1675. doi: 10.1175/ 1520-0426(2000)0172.0.C0;2.

Available at: https://doi.org/10.1175/1520-0426(2000)0172.0.C0;2

This Article is brought to you for free and open access by the Graduate School of Oceanography at DigitalCommons@URI. It has been accepted for inclusion in Graduate School of Oceanography Faculty Publications by an authorized administrator of DigitalCommons@URI. For more information, please contact digitalcommons-group@uri.edu.
} 


\title{
Evaluation of Front Detection Methods for Satellite-Derived SST Data Using In Situ Observations
}

\author{
David S. Ullman and Peter C. CoRnillon \\ Graduate School of Oceanography, University of Rhode Island, Narragansett, Rhode Island
}

(Manuscript received 25 August 1999, in final form 4 January 2000)

\section{ABSTRACT}

\begin{abstract}
Sea surface temperature (SST) fronts detected in Advanced Very High Resolution Radiometer (AVHRR) data using automated edge-detection algorithms were compared to fronts found in continuous measurements of SST made aboard a ship of opportunity. Two histograms (a single-image and a multi-image method) and one gradient algorithm were tested for the occurrence of two types of errors: (a) the detection of false fronts and (b) the failure to detect fronts observed in the in situ data. False front error rates were lower for the histogram methods $(27 \%-28 \%)$ than for the gradient method $(45 \%)$. Considering only AVHRR fronts for which the SST gradient along the ship track was greater than $0.1{ }^{\circ} \mathrm{C} \mathrm{km}^{-1}$, error rates drop to $14 \%$ for the histogram methods and $29 \%$ for the gradient method. Missed front error rates were lower using the gradient method (16\%) than the histogram methods $(30 \%)$. This error rate drops significantly for the histogram methods $(5 \%-10 \%)$ if fronts associated with small-scale SST features $(<10 \mathrm{~km})$ are omitted from the comparison. These results suggest that frontal climatologies developed from the application of automated edge-detection methods to long time series of AVHRR images provide acceptably accurate statistics on front occurrence.
\end{abstract}

\section{Introduction}

Ocean surface thermal fronts are known to be important from the perspectives of both physical and biological oceanography. Strong baroclinic current jets with associated frontal eddies often occur within surface frontal zones. Particularly in coastal seas, frontal regions have been shown to be loci of high primary production with corresponding large standing stocks of phytoplankton (Holligan 1981).

With the increase in the availability of satellite-derived sea surface temperature (SST) data, there has been interest in developing automated methods for detecting fronts in these data. These range from simple methods using a local statistic of the SST field, such as the gradient, variance, or skewness (Cornillon and Watts 1987), to more involved methods, such as cluster-shade algorithms (Holyer and Peckinpaugh 1989) or histogram algorithms (Cayula and Cornillon 1992; Cayula and Cornillon 1995). The Cayula-Cornillon method has been applied to SST imagery for the purpose of generating frontal climatologies by Kahru et al. (1995) in the Baltic Sea, by Ullman et al. (1998) in the North American Great Lakes, and by Ullman and Cornillon (1999) off

Corresponding author address: Dr. David S. Ullman, Graduate School of Oceanography, University of Rhode Island, South Ferry Road, Narragansett, RI 02882.

E-mail: d.ullman@gso.uri.edu the northeast coast of the United States. It has also been applied to global SST fields by I. Belkin et al. (1999, unpublished manuscript). Moore et al. (1997), using a gradient front detection method, investigated the mean path and variability of the polar front in the southern ocean.

When applying automated methods of front detection to satellite imagery, it is important to verify that these techniques are finding real oceanographic fronts and not atmospheric features or artifacts of the processing. Although the use of frontal datasets has thus far been mostly for descriptive purposes, objectively detected fronts could be used for numerical model validation or as data to be assimilated into a model. For these purposes, it is necessary to understand the error statistics of the satellite frontal data (i.e., the degree of confidence that a front exists). One way to approach this problem is to statistically compare the fronts detected in satellite images with those observed in SST data taken from thermosalinographs carried aboard ships in transit. Such a comparison is complicated by the fact that, in the spatial sense, the ship data are one-dimensional while the satellite data are two-dimensional. The greater information content of the satellite data must be accounted for when evaluating error rates of front detection algorithms.

In this paper, we provide a comparison between the SST fronts detected in satellite imagery using both a gradient method and the Cayula-Cornillon method with 


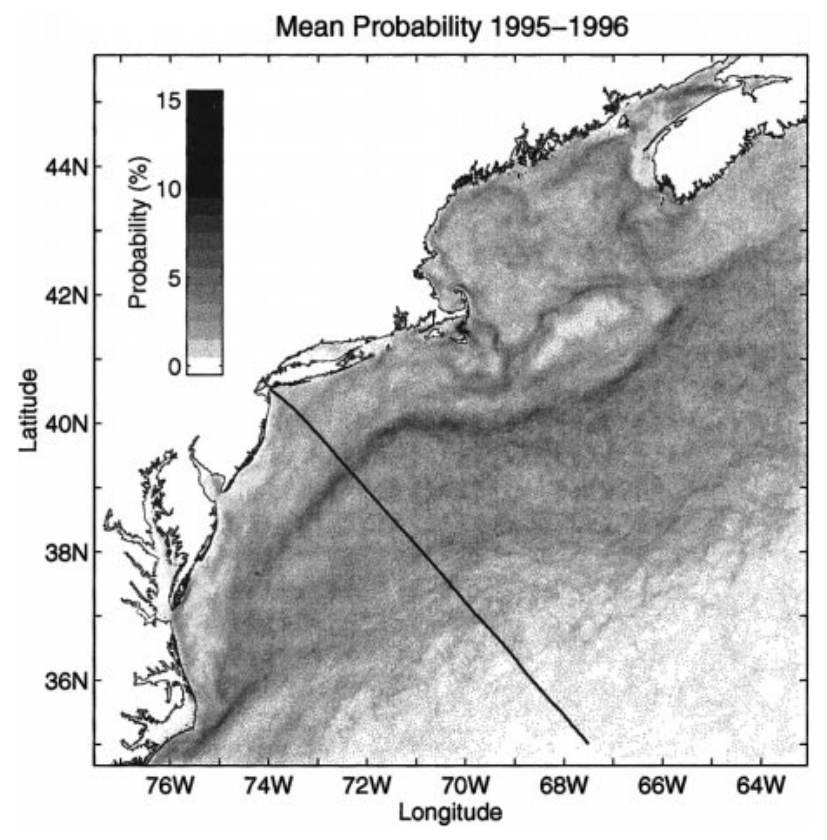

FIG. 1. Mean probability of detecting a front, using the multi-image method, computed over 1995-96. The mean track of the C/V Oleander is shown by the heavy black line.

those detected in continuous temperature measurements made aboard a merchant ship of opportunity in transit between New York and Bermuda. The comparison is broken down into analyses of the rate of detection of false fronts in the satellite data and the rate at which real fronts are missed in the satellite data. The outline of the paper is as follows. Section 2 discusses the data sources and the methods utilized to identify SST fronts in each dataset. Section 3 presents the results of the comparison followed by a summary and conclusions in Section 4

\section{Data and methods}

\section{a. Satellite data processing}

The SST fields for 1995-96, covering the coastal region extending from Cape Hatteras to Nova Scotia (Fig. 1), were derived from Advanced Very High Resolution Radiometer (AVHRR) measurements made aboard the polar orbiting satellites, NOAA-9 and NOAA-14. The SST is calculated from the infrared channels 4 (10.3$11.3 \mu \mathrm{m})$ and $5(11.5-12.5 \mu \mathrm{m})$ using the algorithm developed under the National Oceanic and Atmospheric Administration (NOAA)/National Aeronautics and Space Administration (NASA) Pathfinder program (Smith et al. 1996). Each satellite pass, of which typically 2-4 were available per day, was manually navigated to an accuracy of approximately one pixel $(\sim 1.1$ $\mathrm{km}$ at nadir) and then remapped to an equirectangular projection with a pixel size of $1.2 \mathrm{~km}$ at the image center. Uncertainty in the location of a pixel in the remapped image is estimated to be approximately two pixels. Image SST resolution is $0.125^{\circ} \mathrm{C}$, with an upper bound on the relative (pixel to pixel) error of $0.2^{\circ} \mathrm{C}$ (Ullman and Cornillon 1999). Cloud flagging is accomplished using a multi-image declouding algorithm that is briefly described by Ullman and Cornillon (1999) and is discussed in more detail by T. Mavor et al. (1999, unpublished manuscript).

Detection of fronts in declouded SST images is performed using the method described by Cayula and Cornillon $(1992,1995)$ and with a simple gradient algorithm. A brief description of the Cayula-Cornillon algorithm is given below; further details can be found in the above references. The algorithm consists of a singleimage edge-detection step followed by a step that utilizes edge information from images in the immediate temporal neighborhood to improve the detection of weak fronts and fronts in the vicinity of clouds. In section 3 , the results of both the single-image and multiimage algorithms are compared to the in situ fronts.

The single-image edge-detection procedure uses a series of statistical tests on the temperature field within overlapping $32 \times 32$ pixel windows to identify the presence of fronts within the window. Windows having two clearly identifiable temperature populations (with the means of the populations differing by at least $0.375^{\circ} \mathrm{C}$ ) that are spatially compact are assumed to contain fronts, with pixels at the temperature transition between the two populations marked as edge pixels. Since the edge pixels at this stage do not always form continuous fronts, a local (pixel level) contour following routine, utilizing SST gradient information, is used to connect isolated edge pixels into frontal segments. Segments shorter than 10 pixels are subsequently eliminated from consideration. After the single-image detection step, maps of persistent edges are produced for each image by compositing all edges found by the single-image step in neighboring images (those within 2.5 days of the current image). These persistent edges are thinned and then fed back into the single-image algorithm as additional input to the contour following step.

In addition to the single-image and multi-image algorithms, for the sake of comparison, we also applied a (single image) gradient-type edge-detection algorithm to the AVHRR data. The window-level tests in the Cayula-Cornillon algorithm were replaced in this case by a gradient magnitude test. The Sobel gradient operator (Russ 1995) was used, with all pixels having a gradient magnitude greater than $0.2^{\circ} \mathrm{C} \mathrm{km}^{-1}$ marked as possible edge pixels. The contour following routine was then applied to the image using these pixels. The required CPU usage per image on a DEC/Alpha workstation was approximately $12 \mathrm{~s}$ for the single-image histogram method, $15 \mathrm{~s}$ for the single-image gradient method, and $40 \mathrm{~s}$ for the multi-image histogram method.

\section{b. Ship data processing}

The in situ SST data used in this study were obtained aboard the container ship $\mathrm{C} / \mathrm{V}$ Oleander, a participant 
in the NOAA Volunteer Observing Ship Program. During 1995-96, the ship made approximately weekly trips between Port Elizabeth, New Jersey, and Bermuda. Figure 1 shows the mean ship track superimposed on an image depicting the 2-yr mean probability (1995-96) of detecting a front in AVHRR SST images using the multiimage method. Surface temperature was measured using a continuous flow system with an intake depth of $\sim 5-6$ $\mathrm{m}$. The sampling rate was $15 \mathrm{~s}$ during 1995 and $10 \mathrm{~s}$ during 1996, with a corresponding spatial sampling rate of $\mathrm{O}(100 \mathrm{~m})$. To more closely match the spatial resolution of the AVHRR data, the Oleander temperature data were averaged to $1.2-\mathrm{km}$ spacing alongtrack, with distances measured from West Bank light in lower New York Bay.

Identification of SST fronts in the ship data was accomplished with a gradient-type algorithm. The alongtrack temperature gradient was computed using centered differences. An alongtrack point was identified as a frontal point if the gradient satisfied either of the following criteria:

$$
\begin{aligned}
& \left|\frac{\nabla T}{\nabla s}\right| \geq 0.2^{\circ} \mathrm{C} \mathrm{km}^{-1} \text { or } \\
& \left|\frac{\nabla T}{\nabla s}\right|>0.1^{\circ} \mathrm{C} \mathrm{km}^{-1} \text { and }\left|\frac{\nabla T}{\nabla s}\right|>5\left\langle\left|\frac{\nabla T}{\nabla s}\right|\right\rangle_{70 \mathrm{~km}}
\end{aligned}
$$

where $T$ is thermosalinograph temperature, $s$ is alongtrack distance, and the angle brackets denote an average over approximately $70 \mathrm{~km}$. The thresholds in (1) and (2) were arrived at empirically. Note that a gradient of $0.1^{\circ} \mathrm{C} \mathrm{km}^{-1}$ is equivalent to the smallest resolvable gradient (approximately 1 digital count per pixel) in an SST image. Criterion (2), based on the Fedorov (1986) definition of a front, identifies a front if the alongtrack gradient exceeds this value and is also significantly greater than the average gradient magnitude in the surrounding region.

Implementation of (1) and (2) to an alongtrack temperature series often results in the marking of several adjacent points if the frontal width is of the order of a few kilometers. In these cases, all adjacent frontal points within a region of constant gradient sign were identified. A single front was then located at the mean position of these frontal points.

\section{c. Evaluation of AVHRR front data}

In the following section, the AVHRR front dataset is analyzed in two ways. The rate of detection of false fronts (i.e., fronts not observed in the ship data) and the rate at which in situ fronts are missed are both estimated. False front errors are assessed by searching the ship front set for a front that matches each AVHRR front, while missed front errors are quantified by searching the AVHRR fronts for a front matching each ship front.
We define a matching front as one that occurs within $\Delta t \mathrm{~h}$ and $\Delta x \mathrm{~km}$.

As will be discussed below, the choices of $\Delta t$ and $\Delta x$ are not independent. We selected $\Delta t=6 \mathrm{~h}$ based on the following rationale. The results of Cornillon and Stramma (1985) demonstrated the potential for marked diurnal changes in SST patterns observed in AVHRR imagery. For this reason, we wanted to avoid situations in which satellite and in situ SST fronts were being compared at time lags of $O(12 \mathrm{~h})$. A small value for $\Delta t$ best accomplishes this but at the cost of a reduction in the number of valid matchups. The choice of $6 \mathrm{~h}$ as the cutoff represents a compromise between the desire to minimize diurnal effects and the need to generate a significant number of matchups for statistical purposes. In fact, the error statistics presented in the next section were recomputed (not shown) for different values of $\Delta t$ (and the associated $\Delta x$ discussed below), with only minor differences noted.

The choice of $\Delta x$ is justified as follows. Discrepancies in the position of an SST front can result from 1) AVHRR navigation errors $(\sim 2.5 \mathrm{~km}), 2)$ propagation of SST features in time [ $\sim 5 \mathrm{~km}$ in $6 \mathrm{~h}$, using a propagation speed of $20 \mathrm{~km} \mathrm{day}^{-1}$ (see Lee and Cornillon 1996)], and 3) slope of frontal interfaces giving rise to offsets in the position of the front at the surface and at the depth of the ship intake $(\sim 5 \mathrm{~km}$ using a slope of $10^{-3}$ and sensor depth of $5 \mathrm{~m}$ ). Combining these errors quadratically gives $\Delta x=7.5 \mathrm{~km}$. An independent test of this scale was performed using the data. For each AVHRR (in situ) front, the distance to the nearest in situ (AVHRR) front detected within $\Delta t$ h was computed. Figure 2 presents histograms of the number of matching fronts as a function of the separation distance. At large distances, the number of matches appears to approach a constant value (estimated to be approximately 5 per $2-\mathrm{km}$ bin). Taking this value as the "noise" level in the matchup procedure, approximately $15 \%$ of the fronts deemed matching using $\Delta x=7.5 \mathrm{~km}$ are likely to be incorrectly classified as such, suggesting that the choice of $\Delta x$ is reasonable.

\section{Results}

\section{a. Alongtrack distribution of fronts}

Before discussion of the error rate statistics, we provide in this section an overview of the frontal data. The distribution of SST fronts as a function of distance alongtrack (from New York) in both the thermosalinograph and AVHRR data (using the multi-image method) is shown in Fig. 3. For the latter fronts, only those fronts intersecting a ship track during the periods that the ship was in transit are shown. Peak detection of fronts in the ship data (Fig. 3a) occurs in two zones, centered at 200 and $400 \mathrm{~km}$ from New York. These correspond, respectively, to the shelfbreak frontal zone 
(a) AVHRR Fronts, Closest Match

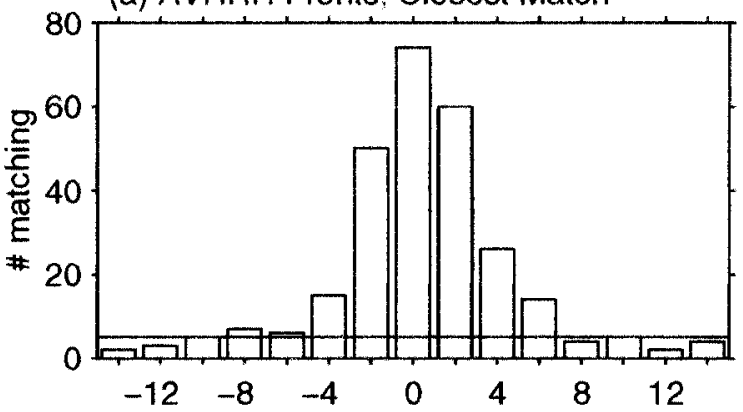

(b) Oleander Fronts, Closest Match

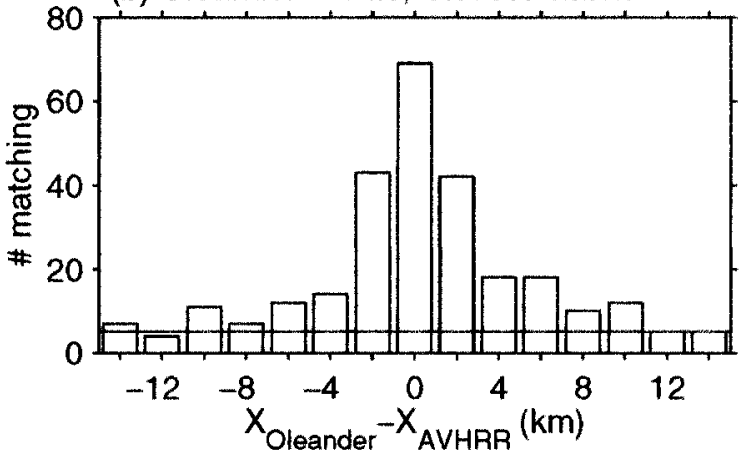

FIG. 2. Histograms of the distance to the (a) nearest matching Oleander front for each AVHRR front (multi-image method) and (b) nearest matching AVHRR front for each Oleander front. The solid line represents the level of five matches per 2-km bin.

and the north wall of the Gulf Stream. In the AVHRR data (Fig. 3b), only the shelfbreak peak is apparent.

An analysis of the number of fronts detected as a function of the degree to which $32 \times 32$ pixel windows were clear (not shown) indicates that $90 \%$ of all AVHRR fronts detected with the multi-image method along the Oleander track are found within windows that are greater than $65 \%$ clear. Figure 3c, depicting the fraction of AVHRR windows that are greater than $65 \%$ clear, shows a general offshore decrease, with a minimum just offshore of the Gulf Stream $(\sim 500 \mathrm{~km})$. Using the distribution in Fig. 3c, the histogram of detected AVHRR fronts can be corrected to obtain the number of fronts that would have been seen if all windows along the Oleander track were at least $65 \%$ clear. An estimate of the expected number of AVHRR fronts, assuming totally clear viewing, is given by the product of the ship front histogram and the ratio of the number of AVHRR images (1281) to the number of Oleander trips (135). The ratio of the corrected to the expected number of fronts in AVHRR images is shown in Fig. 3d. The mean value is approximately 0.7 , indicating an overall underestimation in the number of fronts detected by the multiimage method relative to the ship data algorithm. From 250 to $450 \mathrm{~km}$ (Gulf Stream north wall), the ratio of observed to expected fronts (Fig. 3d) is significantly lower than the average, suggesting that in this region,
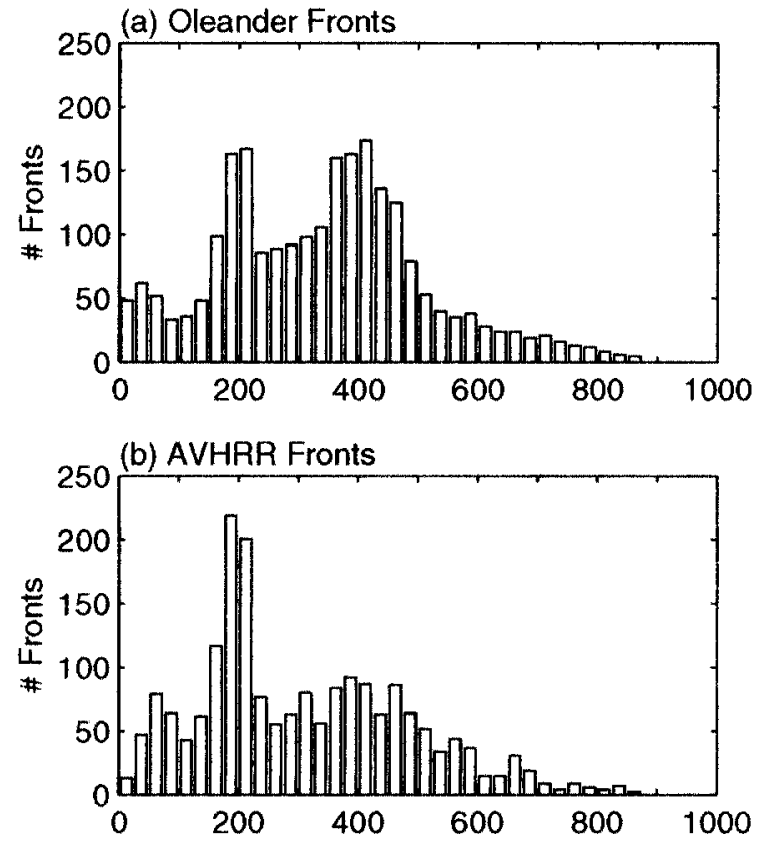

(c) Fraction of Windows $>65 \%$ clear

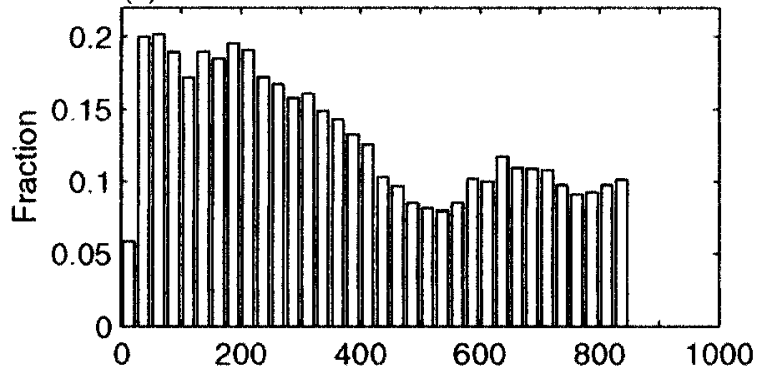

(d) Corrected \# AVHRR / \# Expected

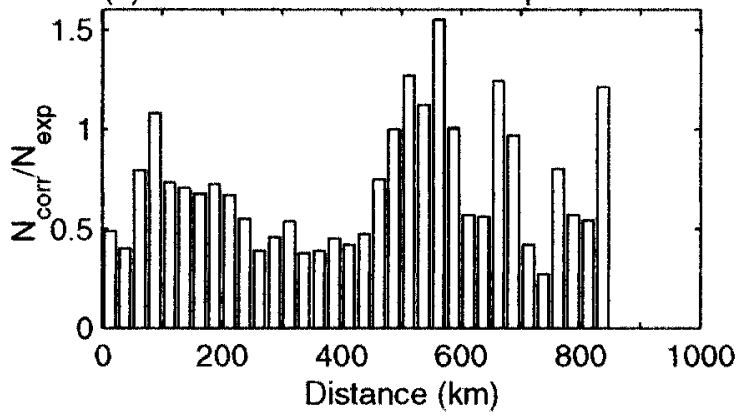

FIG. 3. (a) Histogram of the number of fronts detected in the Oleander thermosalinograph data as a function of the distance from New York for 1995-96. (b) Similar histogram for fronts found in the AVHRR images using the multi-image method. (c) Fraction of $32 \times$ 32 pixel windows that were greater than $65 \%$ clear. (d) Ratio of the corrected number of AVHRR fronts (the number that would have been seen if all windows were at least $65 \%$ clear) to the number of fronts expected based on the Oleander statistics of a). 
TABLE 1. False front statistics for fronts found in AVHRR data.

\begin{tabular}{|c|c|c|c|c|c|}
\hline AVHRR method & $\begin{array}{l}\text { Threshold } \\
\left({ }^{\circ} \mathrm{C} \mathrm{km}^{-1}\right)\end{array}$ & Total & No. matching & No. not matching & $\begin{array}{l}\text { Percent error } \\
\quad \text { (false) }\end{array}$ \\
\hline \multicolumn{6}{|l|}{ I } \\
\hline Multi-image & 0 & 350 & 255 & 95 & 27 \\
\hline Single-image & 0 & 285 & 205 & 80 & 28 \\
\hline Gradient & 0 & 960 & 530 & 430 & 45 \\
\hline \multicolumn{6}{|l|}{ II } \\
\hline Multi-image & 0.1 & 237 & 204 & 33 & 14 \\
\hline Single-image & 0.1 & 190 & 164 & 26 & 14 \\
\hline Gradient & 0.1 & 520 & 371 & 149 & 29 \\
\hline
\end{tabular}

fronts are missed by the multi-image method at an even higher rate. We believe that this results from the behavior of the declouding algorithm. Pixels in the vicinity of transient frontal zones, such as the Gulf Stream, are often flagged as cloudy in an otherwise clear scene. Since the number of frontal pixels is a small fraction of the total number of pixels in a window, the effect on the fraction clear (Fig. 3c) is negligible. The reason that a similar drop in front detection is not observed for the shelfbreak front is that this front experiences less movement than the Gulf Stream front, and hence the temperature discontinuity has less effect on the cloudscreening algorithm.

\section{b. False front error rate}

The first validation issue to be addressed is the estimation of the rate of commission of false front errors. For a given AVHRR front, a matching in situ front is one that is found within $\Delta x \mathrm{~km}$ alongtrack and $\Delta t \mathrm{~h}$ of

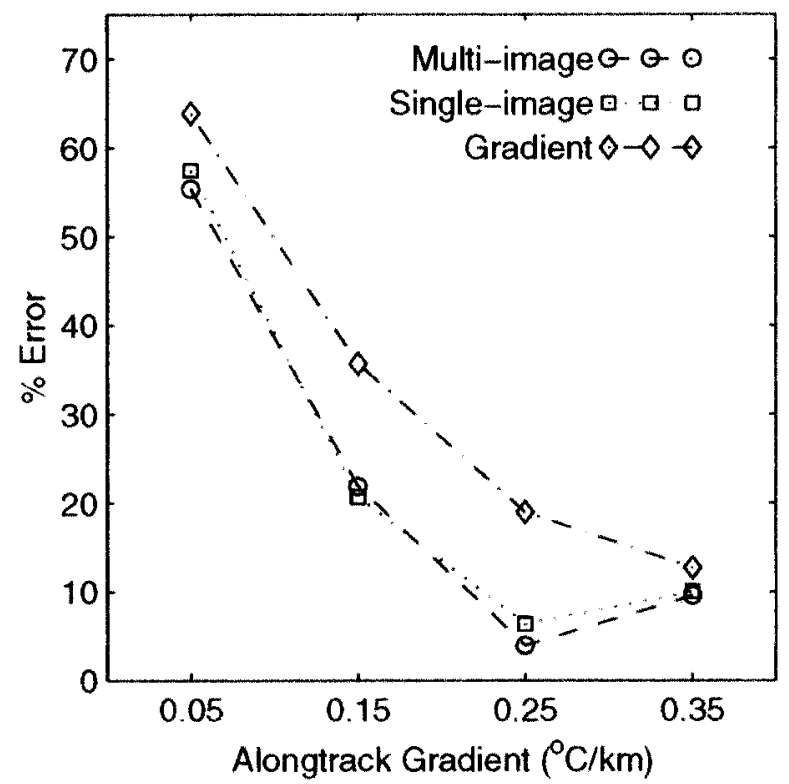

FIG. 4. False front error rate as a function of the alongtrack SST gradient magnitude computed from AVHRR images. Error rates are computed in bins of $0.1^{\circ} \mathrm{C} \mathrm{km}^{-1}$ and plotted at the bin centers. the AVHRR front. Conversely, if the ship was at the location of an AVHRR front within $\Delta t$ h of the AVHRR image time and a front was not found in the ship data, then that AVHRR front was labeled as nonmatching. If the ship did not pass the location of an AVHRR front within this time period, no comparison could be made.

The upper part of Table 1 (I) summarizes the results of this comparison for the three AVHRR edge-detection algorithms considered. The rate of commission of false front errors is $27 \%-28 \%$ for the multi-image and singleimage histogram methods and $45 \%$ for the gradient method. The high error rate is partly due to the fact that a front oriented nearly parallel to and crossing the ship track is likely to be undetectable in thermosalinograph data because the alongtrack SST gradient would be small. For each AVHRR front, the gradient in the alongtrack direction was estimated from the AVHRR SST by averaging the gradient within a $5 \times 5$ pixel box centered on the intersection point between the front and the ship track. Figure 4 shows the (false front) error rate as a function of the alongtrack SST gradient magnitude (in $0.1^{\circ} \mathrm{C} \mathrm{km}^{-1}$ bins). For gradient values between $0^{\circ}$ and $0.1^{\circ} \mathrm{C} \mathrm{km}^{-1}$, the error rate is greater than $40 \%$ for all methods but falls rapidly with increasing gradient magnitude.

The classification of each AVHRR front was adjusted to account for the angle between the ship track and the direction of the SST gradient. The AVHRR fronts for which the estimated alongtrack gradient magnitude was less than $0.1^{\circ} \mathrm{C} \mathrm{km}^{-1}$ [the value of threshold (2) in the definition of an in situ front] were reclassified into the "no comparison" category, since these would be undetectable in the ship data using the front criterion (2). Statistics for the comparison, using this more restrictive definition of a valid matchup, are shown in the lower part of Table 1 (II). For the histogram methods, the adjusted error rate is $14 \%$, and for the gradient method, it is $29 \%$. Comparison of the matchup statistics for a given method with and without application of the gradient threshold shows that a significant number of matching fronts [20\%-30\% of the number matching in Table 1 (I)] were found when the alongtrack gradient was less than $0.1^{\circ} \mathrm{C} \mathrm{km}^{-1}$. Some of these may be a result of errors in the matchup procedure, as noted in section 2c. Most are likely the result of the fact that the gradient 
was estimated from the AVHRR images and thus may be in error because of satellite navigation errors, time differences between satellite passes and the ship observations, and atmospheric effects.

\section{c. Missed front error rate}

Next we consider the reverse comparison, namely the determination of the fraction of the fronts observed in the in situ data that are not detected in the AVHRR imagery (missed front errors). For each in situ front, the AVHRR dataset was searched for a matching front using the same $\Delta t$ and $\Delta x$ used in the previous section. Since a given window in an AVHRR image is likely to be sufficiently clear less than $20 \%$ of the time (see Fig. $3 c$ ), many in situ fronts will not be detected in the AVHRR imagery. Accurate estimation of the rate of commission of missed front errors thus requires consideration of the degree to which the region around an in situ front is clearly viewed as well as the masking effect of clouds on the detection of fronts in AVHRR images.

A measure of the clarity of view in the region around an in situ front is the fraction of the pixels within a 40$\mathrm{km}$ square $(33 \times 33$ pixel $)$ window, centered on the front location, that is not cloud covered. This fraction was computed at each front location for each AVHRR image within $\Delta t \mathrm{~h}$ of the time of detection of the front. For a given front, the maximum fraction clear from the set of images within $\Delta t \mathrm{~h}$ was used as the measure of cloudiness.

To independently assess the effect of cloud cover on the retrieval of fronts in AVHRR imagery, a Monte Carlo simulation was performed as follows. The singleimage edge detector was applied to 50 of the clearest images for the period 1985-96. All fronts in these base images that crossed the mean path of the Oleander were considered to be the in situ fronts. Next, a set of 10 cloud masks from randomly selected images was superimposed on each of the 50 images, and the resulting 500 images were analyzed with the single-image edge detector. For each front found in the base images, matching fronts were sought in each of the 10 masked images, and the fraction clear was computed. This allowed estimation of the (missed front) error rate as a function solely of the fraction clear in the masked image. The result is shown by the solid line in Fig. 5. The error rate is approximately $100 \%$ for clear fractions less than about 0.25 and decreases linearly to zero above this value.

The error rate for each of the AVHRR front detection methods is also shown in Fig. 5 as a function of the fraction clear. The histogram methods exhibit qualitatively the same behavior as the Monte Carlo results, namely, the approximately linear decrease of the error rate with increasing fraction clear above a particular threshold. At a given fraction clear, however, the error rates are roughly $30 \%$ higher than the Monte Carlo error

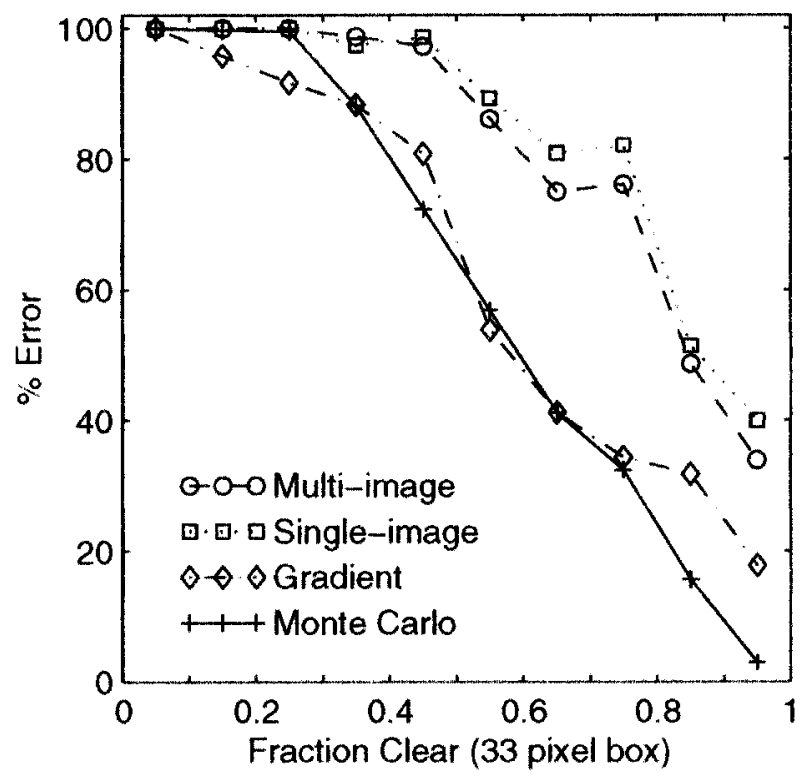

FIG. 5. Missed front error rate as a function of the fraction of a $33 \times 33$ pixel window that is clear in AVHRR images within $6 \mathrm{~h}$ centered on the in situ front location. Also shown is the error rate from the Monte Carlo simulation.

rate, suggesting that in situ fronts are being missed for reasons other than the masking effect of clouds. The error rate for the gradient method is lower than for the histogram methods and displays a qualitatively different dependence on the fraction clear. There is no threshold below which fronts are not found, reflecting the fact that the gradient method is applicable even under very cloudy conditions.

Figure 5 indicates that even with almost completely clear windows in the AVHRR, fronts present in the in situ data are frequently not detected in the AVHRR images. The upper part of Table 2 (I) presents missed front error statistics limited to those in situ fronts for which an AVHRR image was at least $95 \%$ clear within a 33pixel window around the front location. Error rates of $29 \%$ and $34 \%$ are found for the multi-image and singleimage methods, respectively. The gradient method performs better according to this measure, with only $16 \%$ of the in situ fronts missed. In the analysis that follows, we restrict attention to those fronts that correspond to a 95\% clear or higher AVHRR window.

The characteristics of the missed front errors are first investigated in relation to the spatial scale of the SST features associated with the in situ fronts. For each in situ front, the feature scale is defined as the distance from the front to the nearest local SST gradient maximum where the gradient sign is opposite to that at the front. The feature scale (see Fig. 6) is estimated from the in situ data and is a rough measure of the spatial scale of the smaller of the water masses meeting at the front. Note that because it is estimated from the ship data, the feature scale may not be an entirely accurate 
TABLE 2. Missed front statistics for fronts found in Oleander thermosalinograph data for which the fraction clear in an AVHRR image was greater than 0.95 . The threshold in the second column refers to the SST feature scale.

\begin{tabular}{|c|c|c|c|c|c|}
\hline AVHRR method & $\begin{array}{l}\text { Threshold } \\
(\mathrm{km})\end{array}$ & Total & No. matching & $\begin{array}{l}\text { No. not } \\
\text { matching }\end{array}$ & $\begin{array}{l}\text { Percent error } \\
\text { (missed) }\end{array}$ \\
\hline \multicolumn{6}{|l|}{ I } \\
\hline Multi-image & 0 & 116 & 82 & 34 & 29 \\
\hline Single-image & 0 & 116 & 76 & 40 & 34 \\
\hline Gradient & 0 & 116 & 98 & 18 & 16 \\
\hline \multicolumn{6}{|l|}{ II } \\
\hline Multi-image & 10 & 20 & 19 & 1 & 5 \\
\hline Single-image & 10 & 20 & 18 & 2 & 10 \\
\hline Gradient & 10 & 20 & 19 & 1 & 5 \\
\hline
\end{tabular}

representation of the true two-dimensional feature scale. The error rate is shown as a function of the SST feature scale in Fig. 7. The error rate for the histogram methods decreases rapidly with increasing feature spatial scale, with a substantial fraction $(>30 \%)$ of the in situ fronts missed for scales less than $\sim 10 \mathrm{~km}$. The error rate for the gradient algorithm does not decrease as rapidly, indicating that this method is less scale dependent than the histogram method.

To detect a front within a window, the histogram analysis step of the Cayula-Cornillon algorithm requires the spatial extent of the smaller of the two SST populations to be at least $25 \%$ of the window size (Cayula and Cornillon 1992). Given a clear window, a water mass of $10-\mathrm{km}$ width extending across the window represents almost exactly $25 \%$ of the area within a 32-pixel $(\sim 38$ $\mathrm{km}$ ) window. Thus, fronts associated with features at scales smaller than $10 \mathrm{~km}$ separate water masses that are too limited in spatial extent to be detected in the histogram analysis step. However, the contour following step can extend fronts beyond the window in which they were detected (Cayula and Cornillon 1992), making it

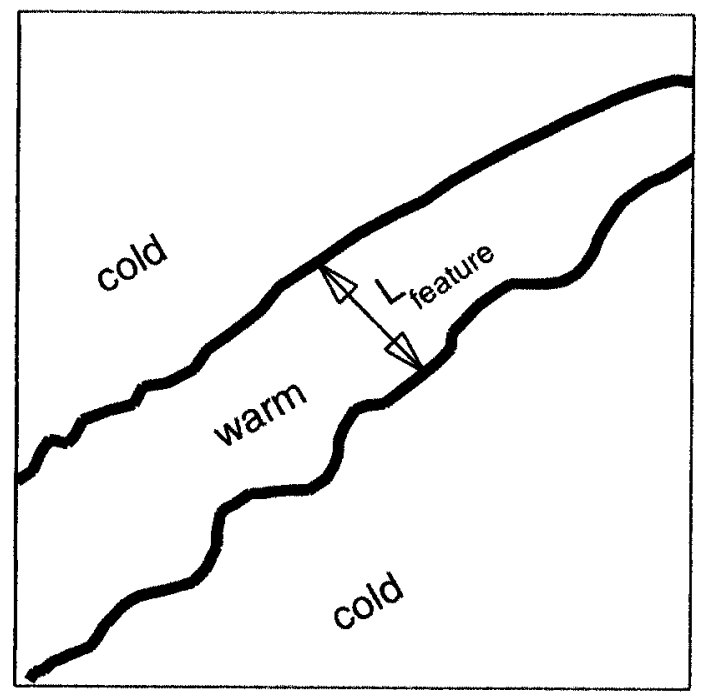

FIG. 6. Diagram showing an example of the SST feature scale $L_{\text {feature }}$. possible to have segments of fronts in windows in which the histogram tests failed to detect a front.

Taking $10 \mathrm{~km}$ as a cutoff for the feature spatial scale, the lower part of Table 2 (II) shows the missed front error rate for all fronts with scales larger than this value. The error rates for the histogram methods drop dramatically to $5 \%$ for the multi-image method and $10 \%$ for the single-image method. Examination of the matchup statistics for the histogram methods with and without the feature scale cutoff shows that many matching fronts were found in the AVHRR images at feature scales less than $10 \mathrm{~km}$. In addition to the fact that roughly $15 \%$ of the matching fronts are likely to be erroneously classified as such (discussed in section 2c), there are several factors that likely contribute to this observation. The first of these is the ability of the contour following step to extend frontal segments into portions of the image in which the histogram analysis failed to detect a front

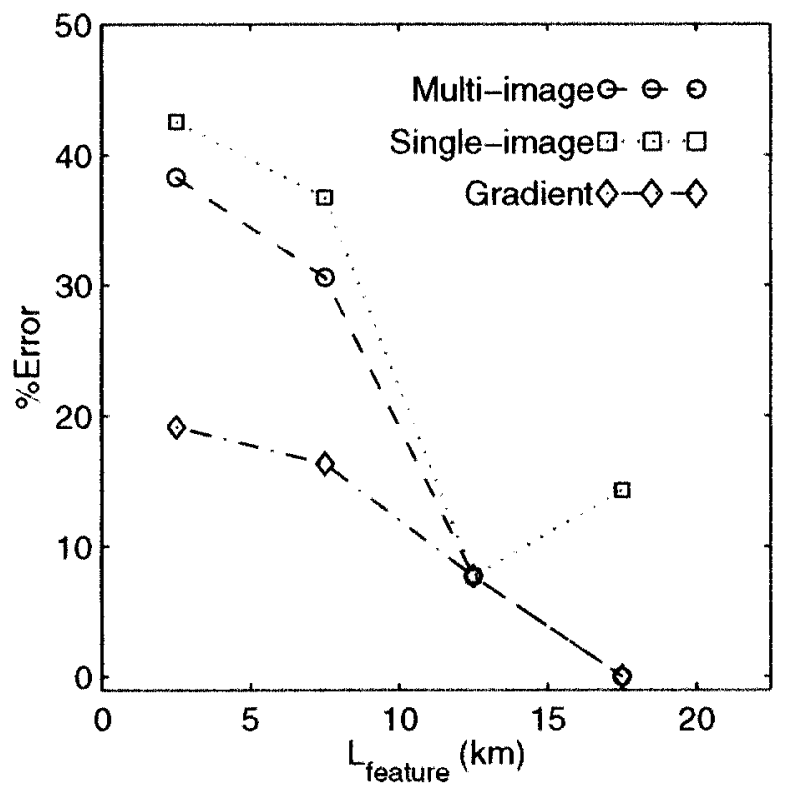

FIG. 7. Missed front error rate as a function of the SST feature scale (see text) for those fronts for which an AVHRR image was at least $95 \%$ clear. Error rates are computed in 5-km bins and plotted at the bin centers. 


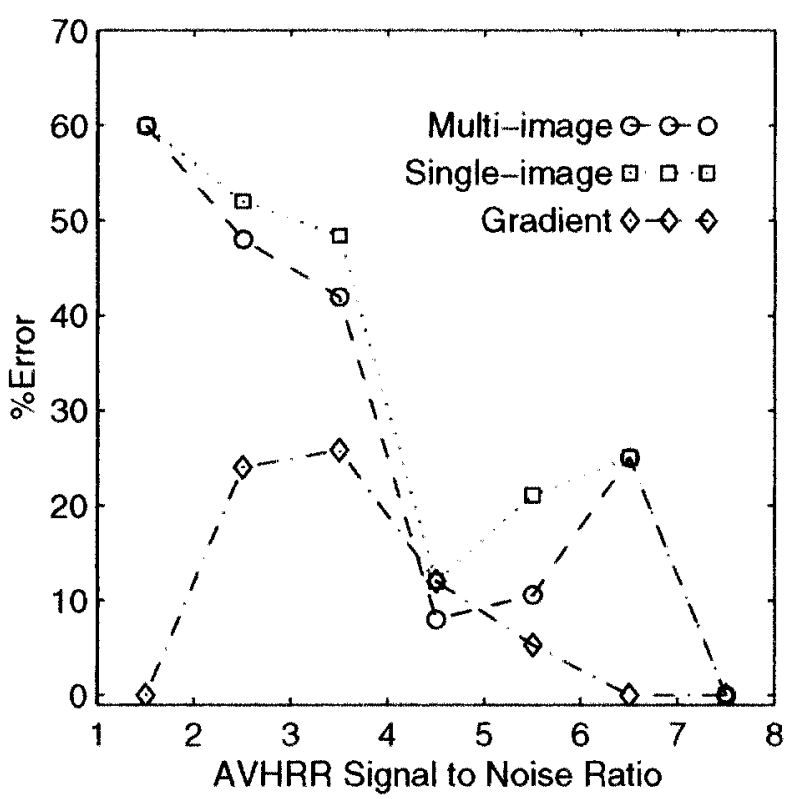

FIG. 8. Missed front error rate as a function of the signal-to-noise ratio in the the AVHRR data along the Oleander track for those fronts for which an AVHRR image was at least 95\% clear. The signal-tonoise ratio is the maximum SST step, within $\pm \Delta x$ of the front, over a distance equal to the in situ front length scale divided by the rootmean-square pixel to pixel SST difference computed over the interval $\pm \Delta x$.

(e.g., because one population was too small). The multiimage step can introduce a similar result, although comparison of the single-image and multi-image results in Table 2 suggest that this effect is small. Finally, as noted above, the feature scale derived from the in situ data is not necessarily a good estimate of the two-dimensional water mass scale either because the ship track was not optimal or because of the presence of complicated multiscale structures that are common, for example, along the north wall of the Gulf Stream [see Fedorov (1986)]. In the latter case, the estimated feature scale would be representative of the small-scale features embedded within the large-scale features, whereas the front-detection algorithm may find a front associated with the underlying large-scale structure.

The detection of fronts in an AVHRR image should depend on the ratio of the magnitude of the SST step to the level of noise in the image. For each in situ front, the corresponding AVHRR SST was examined along the Oleander track to evaluate this ratio. The temperature step was computed using a cross-frontal length scale estimated from the ship data. The maximum SST step over this scale, computed for all AVHRR points within $\pm \Delta x$, was used as the representative step. The SST noise level was computed as the root-mean-square pixel-to-pixel SST difference over the same $\pm \Delta x$ interval. Figure 8 presents the missed front error rate as a function of the AVHRR signal-to-noise ratio. For the histogram edge detectors, the error rate drops abruptly at a signal-to-noise level of about 4. At levels below this value, the noise level is high enough to obscure a real front in about $50 \%$ of the cases while above 4 , the error rate is $\sim 10 \%-20 \%$. The error rate for the gradient method at low signal-to-noise level (1-2) is zero, although there are few fronts detected in this range.

\section{Summary}

This paper has presented the results of a comparison between SST fronts detected in AVHRR imagery and those observed in $2 \mathrm{yr}$ of continuous temperature measurements made aboard a ship of opportunity. Fronts in AVHRR SST images were identified using two histogram methods (Cayula-Cornillon) and a gradient method. Fronts in the ship data were detected using a gradient threshold method. Since errors in the gradients computed from the ship data are much smaller than errors in AVHRR SST due to atmospheric effects, the error rates presented here can be viewed as an assessment of the distorting effect of the atmosphere and the particular edge-detection methodology applied to the SST images.

When estimating the rate of detection of false fronts, it is important to consider the magnitude of the SST gradient along the ship track. This is because an arbitrarily strong front oriented nearly parallel with the ship track will not be detected in the ship data. After eliminating from consideration all AVHRR fronts with alongtrack gradients less than $0.1^{\circ} \mathrm{C} \mathrm{km}^{-1}$, the false front error rate was $14 \%$ using either of the histogram methods and $29 \%$ using the gradient method. The observation that the gradient method finds twice as many false fronts suggests that this method is less tolerant of noise in the SST image than are the histogram methods. In fact, visual inspection of AVHRR images and the fronts detected by the various methods showed that the gradient method often found fronts near the edges of clouds where the SST is often noisy.

The rate at which in situ fronts are missed using the AVHRR detection methods is found to be highly dependent on the degree to which the frontal region is obscured by clouds. The masking effect of clouds was estimated with a Monte Carlo simulation using 50 of the clearest images with randomly chosen cloud masks. If the region around a front is at least $95 \%$ clear, then the error rate due to clouds is estimated to be approximately 0. Actual error rates were subsequently computed using only those in situ fronts for which a $95 \%$ clear image was available. Resulting error rates were approximately $30 \%$ using the histogram methods and $16 \%$ using the gradient method. The relatively high missed front errors with the histogram methods appear to result from two effects. The first is the occurrence of small-scale SST features $(<10 \mathrm{~km})$ in the ship data that may not be resolved by the edge detector operating on $32 \times 32$ pixel windows. Restricting attention to largescale features $(>10 \mathrm{~km})$, error rates of $5 \%$ and $10 \%$ are found for the multi-image and single-image histogram 
methods, respectively, and 5\% for the gradient method. The presence of high noise levels in the AVHRR data was also shown to affect the results, with error rates rising significantly at signal-to-noise levels below 4 .

Comparison of AVHRR SST fronts with in situ fronts leads to the conclusion that automated edge-detection algorithms perform well in detecting SST fronts given clear viewing conditions. The Cayula-Cornillon method performs significantly better than the gradient method in that the false front error rate is $50 \%$ lower, while for fronts associated with features with scales $>10 \mathrm{~km}$, the rate at which in situ fronts are missed is approximately equal for the two methods. Using the error rates estimated here allows us to bound the errors on front occurrence probabilities computed from application of the Cayula-Cornillon edge-detection algorithm to large sets of SST images. Using these rates, we estimate that frontal probabilities should be accurate to within about $15 \%$ for fronts associated with SST features with scales greater than $10 \mathrm{~km}$. Frontal climatologies produced using these methods should thus provide reasonably accurate portrayals of the distribution of fronts at these scales.

Acknowledgments. This research was supported by the National Oceanographic and Atmospheric Adminstration's (NOAA) Sea Grant Program (NA66RG0303) and by the National Aeronautics and Space Administration (NASA) through Grant NAG53736. Salary support for P. Cornillon was provided by the State of Rhode Island and Providence Plantations. We would like to acknowledge the work of Tim Mayor of URI for sharing the workload associated with the declouding and edge detection of the AVHRR images and for many fruitful discussions pertaining to this project. Carl Wolfteich carried out the Pathfinder processing of the AVHRR data used in this study. The Oleander data were provided by Glenn Strout from the National Marine Fisheries Service. The software used for image processing was developed by R. Evans, O. Brown, J. Brown, and A. Li of the University of Miami. Their continued support is greatly appreciated. The comments of an anonymous reviewer helped to improve the presentation of the paper.

\section{REFERENCES}

Cayula, J. F., and P. Cornillon, 1992: Edge detection algorithm for SST images. J. Atmos. Oceanic Technol., 9, 67-80.

, and - 1995: Multi-image edge detection for SST images. J. Atmos. Oceanic Technol., 12, 821-829.

Cornillon, P., and L. Stramma, 1985: The distribution of diurnal sea surface warming events in the western Sargasso Sea. J. Geophys. Res., 90, 11 811-11 815.

- and D. R. Watts, 1987: Satellite thermal infrared and inverted echo sounder determinations of the Gulf Stream northern edge. J. Atmos. Oceanic Technol., 4, 712-723.

Fedorov, K. N., 1986: The Physical Nature and Structure of Oceanic Fronts. Springer-Verlag, 333 pp.

Holligan, P. M., 1981: Biological implications of fronts on the northwest European continental shelf. Philos. Trans. Roy. Soc. London, 302A, 547-562.

Holyer, R. J., and S. H. Peckinpaugh, 1989: Edge detection applied to satellite imagery of the oceans. IEEE Trans. Geosci. Remote Sens., 27, 46-56.

Kahru, M., B. Håkansson, and O. Rud, 1995: Distributions of the sea-surface temperature fronts in the Baltic Sea as derived from satellite imagery. Contin. Shelf Res., 15, 663-679.

Lee, T., and P. Cornillon, 1996: Propagation of Gulf Stream meanders between $74^{\circ}$ and $70^{\circ}$ W. J. Phys. Oceanogr., 26, 205-224.

Moore, J. K., M. R. Abbott, and J. G. Richman, 1997: Variability in the location of the Antarctic Polar Front $\left(90^{\circ}-20^{\circ} \mathrm{W}\right)$ from satellite sea surface temperature data. J. Geophys. Res., 102, $27825-27833$.

Russ, J. C., 1995: The Image Processing Handbook. 2d ed. CRC Press, $674 \mathrm{pp}$

Smith, E. A., J. Vazquez, A. Tran, and R. Sumagaysay, 1996: Satellitederived sea surface temperature data available from the NOAA/ NASA Pathfinder Program. Eos, Trans. Amer. Geophys. Union (Electronic Suppl.). [Available online at http://www.agu.org/ eos_elec/95247e.html.]

Ullman, D. S., and P. C. Cornillon, 1999: Satellite-derived sea surface temperature fronts on the continental shelf off the northeast U.S. coast. J. Geophys. Res., 104, 23 459-23 478.

- J. Brown, P. Cornillon, and T. Mavor, 1998: Surface temperature fronts in the Great Lakes. J. Great Lakes Res., 24, 753775 . 